\title{
Encagement of Gold Nanoclusters in Crosslinked Resorcinarene Shells
}

\author{
STEPHEN V. PUSZTAY ${ }^{a}$, ALEXANDER WEI ${ }^{a, *}$, KEVIN B. STAVENS $^{b}$ and RONALD P. ANDRES ${ }^{b}$ \\ ${ }^{a}$ Department of Chemistry, 1393 Brown Building, Purdue University, West Lafayette, IN 47907-1393, USA; ${ }^{b}$ Department of Chemical Engineering, \\ Purdue University, West Lafayette, IN, USA
}

(Received 21 August 2001; In final form 25 October 2001)

\begin{abstract}
Monolayers of resorcinarene tetraene 1 on gold nanoclusters could be crosslinked by olefin metathesis into a nondesorptive shell. Crosslinking conditions were optimized by monitoring olefin metathesis of 1 by ${ }^{1} \mathbf{H}$ NMR spectroscopy and obtaining empirical rate constants. Gel permeation chromatography (GPC) was found to be a useful tool for evaluating the enhanced stability of the encaged nanoparticles; resorcinareneencapsulated nanoparticles which were not subjected to olefin metathesis did not survive GPC analysis, whereas nanoparticles in crosslinked shells were found to be stable.
\end{abstract}

Keywords: Calixarenes; Nanoparticles; Encapsulation; Olefin metathesis

Monolayer-protected nanoclusters [1] have enormous potential as functional materials. Their sizetunable electronic, optical, or magnetic properties can be synergistically coupled with supramolecular chemistry for a wide range of applications such as biological labeling [2,3], chemical sensing [4-6], catalysis [7] and possibly even nanoscale electronics and computing [8-10]. Many of the applications envisioned for nanoparticle-based materials require good control over their surface chemistry. Metal particles, which are not well protected, degrade quickly and irreversibly into poorly defined aggregate structures, so desorption of the passivating surfactant molecules is a critical problem in this regard. For example, it has been shown that thiol molecules on gold exhibit temperature-dependent mobility [11] and undergo place exchange with other thiols in solution [12]. On the other hand, crosslinking or polymerization of the surfactant layer should result in nanoparticles encaged in a nondesorptive shell. The crosslinked shell strategy has been successfully applied to vesicular bilayer membranes [13], polymer micelles [14,15], dendrimers [16], and very recently to gold nanoparticles [17]. In each of these cases, the interiors were removed to create hollow polymer capsules. Here we demonstrate that crosslinking the surfactant layer greatly enhances the robustness of the encaged nanoparticles against desorption and agglomerationinduced degradation.

We have previously demonstrated that calix[4]resorcinarene ethers such as $\mathbf{1}$ can be used to stablize dispersions of gold nanoparticles in hydrocarbon solvents $[18,19]$. Gold nanoclusters ranging from 5 to $25 \mathrm{~nm}$ were generated as aerosols and passed through a $1 \mathrm{mM}$ solution of $\mathbf{1}$ in mesitylene. The distinctively purplish dispersions appeared to be indefinitely stable at room temperature if left undisturbed. However, particles, which were flocculated by an increase in solvent polarity, were poorly redispersible, indicating that the resorcinarenes were readily displaced from the gold surface and offered little protection against particle degradation. Dilution of the resorcinarene concentration from $1 \mathrm{mM}$ to $0.1 \mathrm{mM}$ also resulted in gradual flocculation, demonstrating the facile desorption of $\mathbf{1}$ from the particle surface.

We envisioned using olefin metathesis as a method to stitch the fragile surfactant layer into a robust, nondesorptive shell (see Fig. 1). We reasoned that the resorcinarene coating was sufficiently dense that a significant proportion of the crosslinking would be intermolecular, yielding a polymeric coating of

*Corresponding author. E-mail: alexwei@purdue.edu 

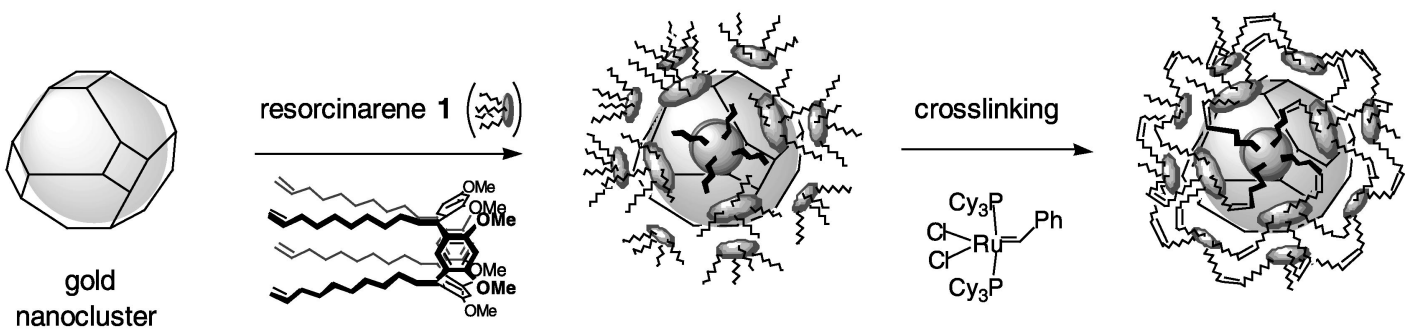

FIGURE 1 Schematic of nanocluster encapsulation by resorcinarene 1, followed by encagement via olefin metathesis.

monolayer thickness [20]. $\dagger$ The ruthenium carbene developed by Grubbs and coworkers has demonstrated to be an extremely mild and versatile catalyst [21], and was therefore expected to be compatible with the weak adsorption of $\mathbf{1}$ on the gold surface.

To ascertain the optimal reaction conditions and proportion of ruthenium catalyst to surfactant, we measured the rate of intramolecular olefin metathesis of $\mathbf{1}$ at different concentrations and catalyst loadings by ${ }^{1} \mathrm{H}$ NMR spectroscopy. $\neq$ These measurements were done in the absence of gold nanoclusters, which would otherwise be present in trace quantities during crosslinking $\left(<10^{11}\right.$ particles $\left./ \mathrm{ml}\right)$. Changes in the $O$-methyl peak distribution were found to be a convenient and straightforward method for monitoring the reaction. The methyl peak singlet assigned to 1 ( $\delta 3.40$ in benzene- $d_{6}$ ) was observed to decrease over time, due to desymmetrization caused by crosslinking of the resorcinarene tails. Data was collected for several different reaction conditions in benzene- $d_{6}$ at room temperature (see Fig. 2). Greater than $90 \%$ consumption of $\mathbf{1}$ was observed only in the case of high catalyst loading $(30 \mathrm{~mol} \%)$, suggesting that the ruthenium species was frequently trapped on partially crosslinked intermediates. $\|$ The consumption rate was determined to be biexponential after taking unreacted $\mathbf{1}$ into account, in accord with earlier mechanistic studies on ruthenium-mediated olefin metathesis [22]. The larger exponential term was used as an effective first-order rate constant; olefin metathesis at high initial reactant concentrations and catalyst loading $\left([1]_{0}=3.6 \mathrm{mM}\right.$ with $30 \mathrm{~mol} \%$ catalyst) yielded a rate constant of $0.52 \mathrm{~min}^{-1}$, corresponding to a reactant half-life of about $1.3 \mathrm{~min}$. This suggested that a reaction time of 5 min would be sufficient to allow substantial crosslinking between resorcinarenes on the nanoparticle surface. Longer reaction times were undesirable, as they may increase the probability of crosslinking between particles.
Freshly prepared dispersions of gold nanoclusters in mesitylene solutions of $\mathbf{1}(1 \mathrm{mM})$ were treated with $30 \mathrm{~mol} \%$ of ruthenium carbene catalyst at room temperature, then quenched after $5 \mathrm{~min}$ with ethyl vinyl ether. The particles' behavior changed substantially after olefin metathesis in at least two respects. First, the nanoclusters no longer formed stable suspensions in mesitylene, but precipitated within a few hours. This is most likely due to the loss of conformational entropy in the resorcinarene chains upon crosslinking, supporting previous conjectures on the importance of chain mobility for effective dispersion $[18,19,23,24]$. Second, the particles were quickly redispersed upon agitation or sonication. This provided some evidence for the enhanced robustness of the crosslinked resorcinarene shells. However, these observations did not affirm the encaged nanoclusters as individually dispersible particles. Transmission electron microscopy (TEM) was also not particularly helpful in this regard, as the great majority of nanoclusters appeared as clumps rather than as isolated particles (see Fig. 3).

Evidence for the robust nature of the encaged nanoclusters was provided by gel permeation chromatographic (GPC) analysis. Nanocluster suspensions in mesitylene were concentrated by approximately an order of magnitude, then loaded onto a crosslinked polystyrene column (Styragel HR4E, Waters) and eluted with chloroform at a flow rate of $0.75 \mathrm{ml} / \mathrm{min}$. Elution of the nanoclusters was monitored at 254 and $540 \mathrm{~nm}$, wavelengths at which gold nanoparticles are known to be strongly absorbing [25]. Nanoclusters encaged in crosslinked resorcinarene shells gave strong signals at both of these wavelengths, and eluted with peakshapes in accord with their size distribution (see Fig. 4). By comparison, nanoclusters encapsulated in noncrosslinked $\mathbf{1}$ did not produce any detectable signals in the same timeframe as the encaged nanoclusters,

\footnotetext{
${ }^{\dagger}$ It should be mentioned that an emulsion polymerization strategy has recently been applied toward nanoparticle encapsulation to give protective coatings ranging in thickness from 2 to $10 \mathrm{~nm}$.

¥The products of intramolecular olefin metathesis of $\mathbf{1}$ were isolated and found to be mostly doubly crosslinked resorcinarene with smaller $(\sim 10-20 \%)$ amounts of singly crosslinked resorcinarene. The yield of the latter product was inversely related to the concentration of the ruthenium catalyst.

${ }^{\uparrow}$ Relatively high concentrations of ruthenium catalyst were also required for efficient crosslinking in other systems; see Refs. [16,17].
} 


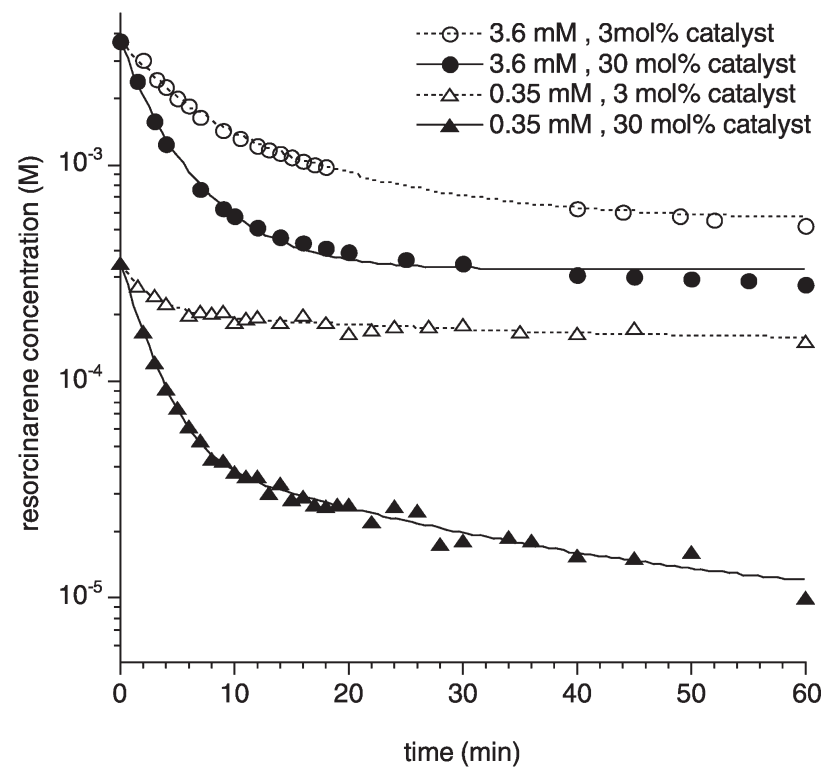

FIGURE 2 Semilogarithmic plot of intramolecular olefin metathesis of $\mathbf{1}$ at various initial reactant concentrations and catalyst loadings, monitored over time by ${ }^{1} \mathrm{H}$ NMR spectroscopy. Measurements were comprised of single scans (acquisition time $=4 \mathrm{~s}$ ) with a minimum delay of $1 \mathrm{~min}$ between measurements. Curves were determined to be biexponential by nonlinear regression analysis.

clearly demonstrating the contrast in robustness between the two systems.

As a point of reference, gold nanoclusters of a similar size range were encapsulated in tetraphosphonite resorcinarene 2 and also analyzed by GPC. The chemisorptive properties of tetraphosphonite 2 are much stronger than that of $\mathbf{1}$, to the extent that encapsulated gold nanoparticles survive GPC analysis [19]. These were found to elute with retention times and peakshapes comparable to those observed for the encaged nanoparticles, a strong indication that the encaged nanoclusters retained their identity

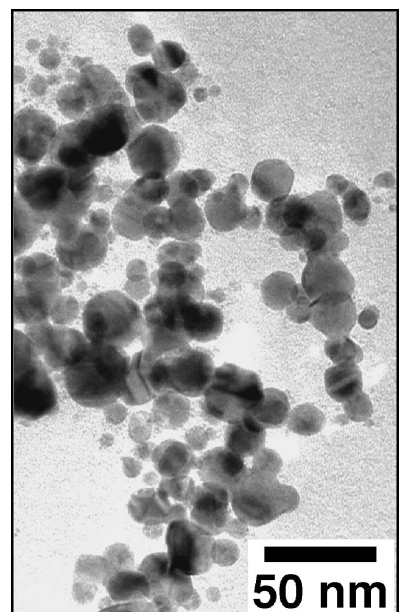

FIGURE 3 Transmission electron micrograph (JEOL 2000 FX, $200 \mathrm{keV}$ ) of resorcinarene-encapsulated nanoclusters subjected to olefin metathesis crosslinking conditions. Samples were obtained by casting a few drops of the mesitylene suspension on a carboncoated TEM grid and allowing the solvent to evaporate.

as discrete particles under appropriately controlled conditions. It is worth mentioning that tetraphosphonite $\mathbf{2}$ is hydrolytically sensitive and therefore has limited chemical stability, whereas the crosslinked resorcinarene shells are expected to be chemically robust under a wide range of processing conditions.

In conclusion, we have demonstrated that resorcinarene-encapsulated gold nanoclusters can be encaged in nondesorptive shells by olefin metathesis. The mechanically robust crosslinked surfactant layer should also be porous, allowing the nanoparticle surface to be accessible to small molecules. This suggests possible applications in size-selective homogeneous catalysis [26] and chemical sensing [4-6].

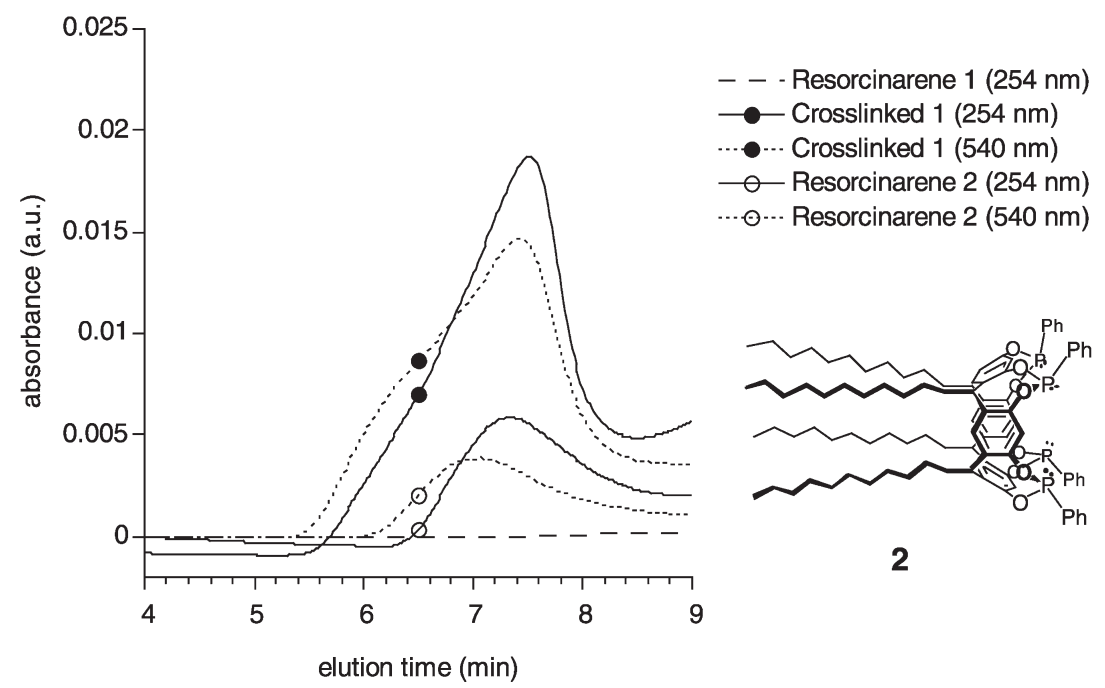

FIGURE 4 GPC analysis of resorcinarene-encapsulated gold nanoclusters. Analysis was performed using a Styragel HR 4E crosslinked polysytrene column (Waters) with $\mathrm{CHCl}_{3}$. Elution was monitored at 254 and $540 \mathrm{~nm}$. 


\section{Acknowledgements}

The authors gratefully acknowledge financial support from the National Science Foundation (BES0086804), DARPA (DAAH04-96-1-0437), and the Research Corporation (RI-0333).

\section{References}

[1] Templeton, A.C., Wuelfing, M.P. and Murray, R.W. (2000), Acc. Chem. Res. 33, 27-36.

[2] Bruchez, Jr, M., Moronne, M., Gin, P., Weiss, S. and Alivisatos, A.P. (1998), Science 281, 2013-2016.

[3] Chan, W.C.W. and Nie, S. (1998), Science 281, 2016-2018.

[4] Wohltien, H. and Snow, A.W. (1998), Anal. Chem. 70, 2856-2859.

[5] Elghanian, R., Storhoff, J.J., Mucic, R.C., Letsinger, R.L. and Mirkin, C.A. (1997), Science 277, 1078-1081.

[6] Wei, A., Kim, B., Sadtler, B. and Tripp, S.L. (2001), ChemPhysChem. 2, 743-745.

[7] Bönnemann, H. and Brijoux, W. (1996) In: Moser, W.R., ed, Advanced Catalysts and Nanostructured Materials (Academic Press, San Diego), pp 165-196.

[8] Andres, R.P., Bielefeld, J.D., Henderson, J.I., Janes, D.B., Kolagunta, V.R., Kubiak, C.P., Mahoney, W.J. and Osifchin, R.G. (1996), Science 273, 1690-1693.

[9] Collier, C.P., Vossmeyer, T. and Heath, J.R. (1998), Annu. Rev. Phys. Chem. 49, 371-404

[10] Ryan, D., Rao, S.N., Rensmo, H., Fitzmaurice, D., Preece, J.A., Wenger, S., Stoddart, J.F. and Zaccheroni, N. (2000), J. Am. Chem. Soc. 122, 6252-6257.
[11] Delamarche, E., Michel, B., Biebuyck, H.A. and Gerber, C (1996), Adv. Mater. 8, 719-729.

[12] Schlenoff, J.B., Li, M. and Ly, H. (1995), J. Am. Chem. Soc. 117, $12528-12536$.

[13] O'Brien, D.F., Armitage, B., Benedicto, A., Bennett, D.E. Lamparski, H.G., Lee, Y.-S., Srisiri, W. and Sisson, T.M. (1998), Acc. Chem. Res. 31, 861-868.

[14] Wooley, K.L. (1997), Chem. Eur. J. 3, 1397-1399.

[15] Bütun, V., Billingham, N.C. and Armes, S.P. (1998), J. Am. Chem. Soc. 120, 12135-12136.

[16] Wendland, M.S. and Zimmerman, S.C. (1999), J. Am. Chem. Soc. 121, 1389-1390.

[17] Wu, M.L., O'Neill, S.A., Brousseau, L.C., McConnell, W.P., Shultz, D.A., Linderman, R.J. and Feldheim, D.L. (2000), Chem. Commun., 775-776.

[18] Stavens, K.B., Pusztay, S.V., Zou, S., Andres, R.P. and Wei, A. (1999), Langmuir 15, 8337-8339.

[19] Wei, A., Stavens, K.B., Pusztay, S.V. and Andres, R.P. (1999), MRS Symp. Proc. Ser. 581, 59-63.

[20] Quaroni, L. and Chumanov, G. (1999), J. Am. Chem. Soc. 121, 10642-10643.

[21] Grubbs, R.H., Miller, S.J. and Fu, G.C. (1995), Acc. Chem. Res. 28, 446-452.

[22] Dias, E.L., Nguyen, S.T. and Grubbs, R.H. (1997), J. Am. Chem. Soc. 119, 3887-3897.

[23] Kim, B., Tripp, S.L. and Wei, A. (2001), J. Am. Chem. Soc. 123, 7955-7956.

[24] Balasubramanian, R., Xu, J., Kim, B., Sadtler, B. and Wei, A. (2001), J. Dispersion Sci. Tech. 22, 485-489.

[25] Kreibig, U. and Vollmer, M. (1995) Optical Properties of Metal Clusters (Springer, New York).

[26] Niu, Y.H., Yeung, L.K. and Crooks, R.M. (2001), J. Am. Chem. Soc. 123, 6840-6846. 